\title{
A solution to dynamic errors-in-variables within system equations
}

\author{
Vahid Mahboub ${ }^{1} \cdot$ Mohammad Saadatseresht $^{1}$. \\ Alireza A. Ardalan ${ }^{1}$
}

Received: 8 December 2016/ Accepted: 22 June 2017/Published online: 10 July 2017

(C) Akadémiai Kiadó 2017

\begin{abstract}
We noticed that if INS data is used as system equations of a Kalman filter algorithm for integrated direct geo-referencing, one encounters with a dynamic errors-invariables (DEIV) model. Although DEIV model has been already considered for observation equations of the Kalman filter algorithm and a solution namely total Kalman filter (TKF) has been given to it, this model has not been considered for system equations (dynamic model) of the Kalman filter algorithm. Thus, in this contribution, for the first time we consider DEIV model for both observation equations and system equations of the Kalman filter algorithm and propose a least square prediction namely integrated total Kalman filter in contrast to the TKF solution of the previous approach. The variance matrix of the unknown parameters are obtained. Moreover, the residuals for all variables are predicted. In a numerical example, integrated direct geo-referencing problem is solved for a GPS-INS system.
\end{abstract}

Keywords Dynamic errors-in-variables $\cdot$ System equations $\cdot$ Integrated total Kalman filter · Direct geo-referencing

\section{Introduction}

Recently, there has been an explosion in the number, type and diversity of system designs and application areas of mobile sensors. The geo-referencing of these systems is one of the main problems. In this problem, one aims to determine the position and attitude of a mobile sensor in a geo-referenced frame. When this information is attained directly by means of

Mohammad Saadatseresht

msaadat@ut.ac.ir

1 School of Surveying and Geospatial Engineering, College of Engineering, University of Tehran,

Tehran, Iran 
measurements from sensors on-board the vehicle the term direct geo-referencing is used (Skaloud 1999). The integration of these data is done during a Kalman filter algorithm (Kalman 1960). For more details on Kalman filter one may refer to Sorenson (1966) and Maybeck (1979). The Kalman filter is essentially a set of mathematical equations that implement a predictor-corrector type estimator that is optimal in the sense that it minimizes the estimated error covariance, when some presumed conditions are met (Welch and Bishop 2001). In the literature, the Kalman filter is derived as either a best predictor (BP) or a best linear predictor (BLP), see e.g. Kalman (1960), Gelb (1974), Sanso (1986). The minimum mean squared error (MMSE) is the criterion which selects the best predictor or estimator.

Observation equations and system equations are two main parts of a dynamic problem. The former is in fact a relation between the observations and time dependent unknown parameters while the latter relates the unknown parameters at an epoch $i$ to an earlier epoch $i-1$. Due to how these two parts are modeled, several linear and non-linear Kalman filters have been proposed. For more information see e.g. Yi (2007). Some filters are as follows: the Sigma Point Kalman Filters (SPKF) (van der Merwe and Wan 2003) or Linear Regression Kalman Filters (LRKF) (Lefebvre et al. 2002), Extended Kalman Filter (EKF) (Jazwinski 1970), the Particle Filters (PF) (Liu and Chen 1998), the Ensemble Kalman Filter (EnKF) (Evensen 1994), Unscented Kalman Filter (UKF) based on unscented transformation (UT) (Julier and Uhlmann 1997) and etc. However, in all of these algorithms, the coefficient matrix of the system equations does not contain random errors. As such an assumption cannot always be guaranteed, we allow random observational errors to enter the respective matrix. In practice, this situation can be seen when we are going to use INS data as the system equations since in such a case, the random observed angular increments and velocity increments measured by gyroscope and accelerator of the INS system, make the coefficient matrix of the system equations noisy.

Note that although Schaffrin and Iz (2008), Schaffrin and Uzun (2011) and Mahboub et al. (2016) considered the case which only the design matrix of the observation equations is random, we solve the problem which both of the coefficient matrix of the observation equations and system equations are corrupted by random noise. Hence in contrast to Schaffrin and Iz (2008) that named their solution total Kalman filter (TKF), we propose an integrated total Kalman filter (ITKF) algorithm.

This paper is organized as follows: in Sect. 2, the DEIV model and the TKF solution proposed by Schaffrin and Iz (2008) are introduced. In Sect. 3, the ITKF algorithm is developed, then, in a later section, a numerical example gives insight into the efficiency of the algorithm proposed. Finally we conclude the paper.

\section{Dynamic errors-in-variables (DEIV) model}

In this section the concepts of dynamic errors-in-variables (DEIV) model are introduced and a TKF solution proposed by Schaffrin and Iz (2008) is given. It must be mentioned that EIV model in its time invariant case i.e. static case has been investigated by several valuable publications. Therefore, we only give some references e.g. Zeng et al. (2015), Zhang et al. (2013), Neitzel (2010), Neitzel and Schaffrin (2016), Snow and Schaffrin (2012), Shen et al. (2011), Schaffrin et al. (2014), Schaffrin and Felus (2008), Mahboub (2012, 2014, 2016), Mahboub et al. (2012, 2015), Mahboub and Sharifi (2013a, b), Paláncz and Awange (2012), Amiri-simkooei and Jazaeri (2012), Fang (2011, 2013, a, b c, 2015), 
Fang et al. (2015, 2016), Lu et al. (2014), Zhou and Fang (2015) and Fang and Wu (2015) etc. In the rest of this paper we define these two parts for a DEIV model. Observation equations is given as follows:

$$
\underline{y}_{i}=\left(A_{i}-\underline{E}_{A_{i}}\right) \underline{x}_{i}+\underline{e}_{i}
$$

In the above equations $\underline{y}_{i}$ is the $\mathrm{m} \times 1$ random observation vector, $\underline{e}_{i}$ is the $\mathrm{m} \times 1$ vector of observational noise, $A_{i}$ is the $\mathrm{m} \times \mathrm{n}$ coefficient matrix of input variables (observed), $\underline{E}_{A_{i}}$ is the corresponding the $\mathrm{m} \times \mathrm{n}$ matrix of random noise, $\underline{x}_{i}$ is the $\mathrm{n} \times 1$ random parameter vector (time dependent unknowns). The following equation represents system equations which is also called dynamic model. It relates the unknown parameters at an epoch $i$ to an earlier epoch $i-1$.

$$
\underline{x}_{i}=\left(\Phi_{i}-\underline{E}_{\Phi_{i}}\right) x_{i-1}+f_{i}+\underline{u}_{i}
$$

$\Phi_{i}$ is the transition matrix $\underline{E}_{\Phi_{i}}$ is the corresponding the $\mathrm{n} \times \mathrm{n}$ matrix of random noise and $\underline{u}_{i}$ is the random system noise, $f_{i}$ is an independent time variable function and underlining (_) indicates random variables. The random noise of the transition matrix is our main problem in this paper. We also assume that the state vector is observed at an initial (previous) epoch:

$$
\underline{x}_{i-1}=x_{i-1}+\underline{e}_{i-1}^{0}
$$

Here, $\underline{e}_{i-1}^{0}$ is the random noise at the first epoch. Equations (1)-(3) represent the functional model of the DEIV model in this paper. We also define the corresponding stochastic model as follows:

$$
\left[\begin{array}{c}
\underline{e}_{i} \\
\underline{e}_{A_{i}}=\operatorname{vec}\left(\underline{E}_{A_{i}}\right) \\
\underline{u}_{i} \\
\underline{e}_{\Phi_{i}}= \\
\operatorname{vec}\left(\underline{E}_{\Phi_{i}}\right) \\
\underline{e}_{i-1}^{0}
\end{array}\right] \sim\left(\left[\begin{array}{l}
0 \\
0 \\
0 \\
0 \\
0
\end{array}\right],\left[\begin{array}{ccccc}
Q_{y_{i}} & 0 & 0 & 0 & 0 \\
0 & Q_{A_{i}} & 0 & 0 & 0 \\
0 & 0 & \theta_{i} & 0 & 0 \\
0 & 0 & 0 & Q_{\Phi_{i}} & 0 \\
0 & 0 & 0 & 0 & \sum_{i-1}^{0}
\end{array}\right]\right)
$$

where $Q_{y_{i}}, \theta_{i}, \sum_{i-1}^{0}, Q_{A_{i}}$ and $Q_{\Phi_{i}}$ are the corresponding dispersion matrixes of the observation vector, system equations, the observed unknown parameters at an initial epoch, the random coefficient matrix $\underline{E}_{A_{i}}$ and the random coefficient matrix $\underline{E}_{\Phi_{i}}$. Schaffrin and Iz (2008) supposed that $\underline{E}_{\Phi_{i}}=0, f_{i}=0, Q_{A_{i}}=I_{n} \otimes Q_{y_{i}}$ and set the following target function:

$$
\begin{aligned}
\Phi\left(\underline{e}_{i}, \underline{e}_{A_{i}}, \lambda_{i}, \mu_{i}\right):= & \left(\underline{e}_{i}^{T} Q_{y_{i}}^{-1} \underline{e}_{i}+\underline{e}_{A_{i}}^{T}\left(I_{n} \otimes Q_{y_{i}}\right)^{-1} \underline{e}_{A_{i}}\right. \\
& +\left(\underline{u}_{i}-\Phi_{i} \underline{e}_{i-1}^{0}\right)^{T}\left(\theta_{i}+\Phi_{i} \sum_{i-1}^{0} \Phi_{i}^{T}\right)^{-1}\left(\underline{u}_{i}-\Phi_{i} \underline{e}_{i-1}^{0}\right) \\
& +2 \lambda_{i}^{T}\left(y_{i}-A_{i}\left(\underline{u}_{i}-\Phi_{i} \underline{e}_{i-1}^{0}+\check{x}_{i}\right)+\left(\left(\underline{u}_{i}-\Phi_{i} \underline{e}_{i-1}^{0}+\check{x}_{i}\right)^{T} \otimes I_{m}\right) \underline{e}_{A_{i}}-\underline{e}_{i}\right)
\end{aligned}
$$

where $\lambda_{i}$ is a $m \times 1$ vector of Lagrange multipliers. They obtained the following leastsquares prediction and named it total Kalman filter (TKF):

$$
\tilde{x}_{i}=\check{x}_{i}+\left(\theta_{i}+\Phi_{i} \sum_{i-1}^{0} \Phi_{i}^{T}\right)\left[A_{i}^{T} \hat{\lambda}_{i}+\tilde{x}_{i}\left(\hat{\lambda}_{i}^{T} Q_{y_{i}} \hat{\lambda}_{i}\right)\right]
$$

where $\check{x}_{i}$ and $\hat{\lambda}_{i}$ are given as follows: 


$$
\begin{gathered}
\hat{\lambda}_{i}=\left(Q_{y_{i}}\right)^{-1}\left(y_{i}-A_{i} \tilde{x}_{i}\right)\left(1+\tilde{x}_{i}^{T} \tilde{x}_{i}\right)^{-1} \\
\check{x}_{i}=\Phi_{i} \tilde{x}_{i-1} .
\end{gathered}
$$

As the assumption $\underline{E}_{\Phi_{i}}=0$ may not be always correct in particular when the system Eqs. (2) are produced by INS data, in the next section we obtain a new solution to this problem.

\section{Integrated total Kalman filter (ITKF)}

In this section we solve the DEIV model given by Eqs. (1)-(4). Since we suppose that both of the coefficient matrixes in the observation equations and system equations are noisy i.e. $\underline{E}_{\Phi_{i}} \neq 0$ and $\underline{E}_{A_{i}} \neq 0$, we call our least-squares prediction "integrated total Kalman filter $(I T K F)$ ". If we want to use condition equations for our optimization, we require combining Eqs. (1)-(3). For this aim, first we insert Eq. (3) into Eq. (1) as follows:

$$
\underline{x}_{i}=\left(\Phi_{i}-\underline{E}_{\Phi_{i}}\right)\left(\underline{x}_{i-1}-\underline{e}_{i-1}^{0}\right)+f_{i}+\underline{u}_{i}
$$

Then we put Eq. (9) into Eq. (1):

$$
\underline{y}_{i}=\left(A_{i}-\underline{E}_{A_{i}}\right)\left(\left(\Phi_{i}-\underline{E}_{\Phi_{i}}\right)\left(\underline{x}_{i-1}-\underline{e}_{i-1}^{0}\right)+f_{i}+\underline{u}_{i}\right)+\underline{e}_{i}
$$

Eventually we can set the following least-squares target function:

$$
\begin{aligned}
\Phi\left(\underline{e}_{i}, \underline{e}_{A_{i}}, \lambda_{i}, \underline{e}_{\Phi_{i}}, \underline{u}_{i}, \underline{e}_{i-1}^{0}\right):= & \underline{e}_{i}^{T} Q_{y_{i}}^{-1} \underline{e}_{i}+\underline{e}_{A_{i}}^{T} Q_{A_{i}}^{-1} \underline{e}_{A_{i}}+\underline{u}_{i}^{T} \theta_{i}^{-1} \underline{u}_{i}+\underline{e}_{\Phi_{i}}^{T} Q_{\Phi_{i}}^{-1}+\underline{e}_{i-1}^{0}\left(\sum_{i-1}^{0}\right)^{-1} \underline{e}_{i-1}^{0} \\
& +2 \lambda_{i}^{T}\left(y_{i}-\underline{e}_{i}-\left(A_{i}-\underline{E}_{A_{i}}\right)\left(\left(\Phi_{i}-\underline{E}_{\Phi_{i}}\right)\left(\underline{x}_{i-1}-\underline{e}_{i-1}^{0}\right)+f_{i}+\underline{u}_{i}\right)\right)
\end{aligned}
$$

Note that in contrast to target function of Eq. (5) proposed by Schaffrin and Iz (2008), the target function given by Eq. (11) can produce the predicted residuals of all random observed variables. In Schaffrin and Iz (2008) the quantities $\tilde{u}_{i}$ an $\tilde{e}_{i-1}^{0}$ were not predicted. For optimization, if tildas $(\sim)$ indicate predicted vectors and hats $\left({ }^{\sim}\right)$ denote estimated ones the following necessary conditions must hold:

$$
\begin{gathered}
\frac{\partial \Phi}{\partial \tilde{e}_{i}} \mid \tilde{e}_{i}, \tilde{e}_{A_{i}}, \hat{\lambda}_{i}, \tilde{e}_{\Phi_{i}}, \tilde{u}_{i}, \tilde{e}_{i-1}^{0}=2\left(Q_{y_{i}}^{-1} \tilde{e}_{i}-\hat{\lambda}_{i}\right)=0 \\
\frac{\partial \Phi}{\partial \tilde{e}_{A_{i}}} \mid \tilde{e}_{i}, \tilde{e}_{A_{i}}, \hat{\lambda}_{i}, \tilde{e}_{\Phi_{i}}, \tilde{u}_{i}, \tilde{e}_{i-1}^{0}=2\left(\left(\left(\Phi_{i}-\tilde{E}_{\Phi_{i}}\right)\left(\underline{x}_{i-1}-\tilde{e}_{i-1}^{0}\right)+f_{i}+\tilde{u}_{i}\right) \otimes I_{m}\right) \hat{\lambda}_{i}+2 Q_{A_{i}}^{-1} \tilde{e}_{A_{i}}=0 \\
=0 \\
\frac{\partial \Phi}{\partial \tilde{e}_{\Phi_{i}}} \mid \tilde{e}_{i}, \tilde{e}_{A_{i}}, \hat{\lambda}_{i}, \tilde{e}_{\Phi_{i}}, \tilde{u}_{i}, \tilde{e}_{i-1}^{0}=2\left(\left(\underline{x}_{i-1}-\tilde{e}_{i-1}^{0}\right) \otimes\left(A_{i}-\tilde{E}_{A_{i}}\right)^{T}\right) \hat{\lambda}_{i}+2 Q_{\Phi_{i}}^{-1} \tilde{e}_{\Phi_{i}}=0 \\
\frac{\partial \Phi}{\partial \tilde{u}_{i}} \mid \tilde{e}_{i}, \tilde{e}_{A_{i}}, \hat{\lambda}_{i}, \tilde{e}_{\Phi_{i}}, \tilde{u}_{i}, \tilde{e}_{i-1}^{0}=-2\left(A_{i}-\tilde{E}_{A_{i}}\right)^{T} \hat{\lambda}_{i}+2 \theta_{i}^{-1} \tilde{u}_{i}=0
\end{gathered}
$$




$$
\begin{aligned}
\frac{\partial \Phi}{\partial \tilde{e}_{i-1}^{0}} \mid \tilde{e}_{i}, \tilde{e}_{A_{i}}, \hat{\lambda}_{i}, \tilde{e}_{\Phi_{i}}, \tilde{u}_{i}, \tilde{e}_{i-1}^{0}=2\left(\Phi_{i}-\tilde{E}_{\Phi_{i}}\right)^{T}\left(A_{i}-\tilde{E}_{A_{i}}\right)^{T} \hat{\lambda}_{i}+2\left(\Sigma_{i-1}^{0}\right)^{-1} \tilde{e}_{i-1}^{0}=0 \\
\begin{aligned}
\frac{\partial \Phi}{\partial \hat{\lambda}_{i}} \mid \tilde{e}_{i}, \tilde{e}_{A_{i}}, \hat{\lambda}_{i}, \tilde{e}_{\Phi_{i}}, \tilde{u}_{i}, \tilde{e}_{i-1}^{0} & =2\left(y_{i}-\tilde{e}_{i}-\left(A_{i}-\underline{E}_{A_{i}}\right)\left(\left(\Phi_{i}-\tilde{E}_{\Phi_{i}}\right)\left(\underline{x}_{i-1}-\underline{e}_{i-1}^{0}\right)+f_{i}+\tilde{u}_{i}\right)\right) \\
& =0 .
\end{aligned}
\end{aligned}
$$

$\tilde{e}_{i}$ and $\tilde{e}_{A_{i}}$ can be obtained from Eqs. (12) and (13) as follows

$$
\begin{gathered}
\tilde{e}_{i}=Q_{y_{i}} \hat{\lambda}_{i} \\
\tilde{e}_{A_{i}}=-Q_{A_{i}}\left(\left(\left(\Phi_{i}-\tilde{E}_{\Phi_{i}}\right)\left(\underline{x}_{i-1}-\tilde{e}_{i-1}^{0}\right)+f_{i}+\tilde{u}_{i}\right) \otimes I_{m}\right) \hat{\lambda}_{i}=-Q_{A_{i}} R_{i} \hat{\lambda}_{i}
\end{gathered}
$$

Equations (14) and (15) immediately lead to

$$
\begin{gathered}
\tilde{e}_{\Phi_{i}}=-Q_{\Phi_{i}}\left(\left(\underline{x}_{i-1}-\tilde{e}_{i-1}^{0}\right) \otimes\left(A_{i}-\tilde{E}_{A_{i}}\right)^{T}\right) \hat{\lambda}_{i}=-Q_{\Phi_{i}} S_{i} \hat{\lambda}_{i} . \\
\tilde{u}_{i}=\theta_{i}\left(A_{i}-\tilde{E}_{A_{i}}\right)^{T} \hat{\lambda}_{i}
\end{gathered}
$$

Equation (16) gives $\tilde{e}_{i-1}^{0}$ as follows:

$$
\tilde{e}_{i-1}^{0}=-\Sigma_{i-1}^{0}\left(\left(A_{i}-\tilde{E}_{A_{i}}\right)\left(\Phi_{i}-\tilde{E}_{\Phi_{i}}\right)\right)^{T} \hat{\lambda}_{i}
$$

Eventually by inserting Eqs. (18)-(22) into Eq. (17), the vector of Lagrange multipliers $\hat{\lambda}_{i}$ can be estimated as follows:

$$
\begin{aligned}
y_{i}- & Q_{y_{i}} \hat{\lambda}_{i}-\left(A_{i}-\tilde{E}_{A_{i}}\right) \theta_{i}\left(A_{i}-\tilde{E}_{A_{i}}\right)^{T} \hat{\lambda}_{i}-S_{i}^{T} Q_{\Phi_{i}} S_{i} \hat{\lambda}_{i} \\
& -\left(A_{i}-\tilde{E}_{A_{i}}\right) \Sigma_{i-1}^{0}\left(\left(A_{i}-\tilde{E}_{A_{i}}\right)\left(\Phi_{i}-\tilde{E}_{\Phi_{i}}\right)\right)^{T} \hat{\lambda}_{i}-A_{i} \Phi_{i} \underline{x}_{i-1} \\
- & \left(\left(\Phi_{i} \underline{x}_{i-1}+f_{i}\right) \otimes I_{m}\right) Q_{A_{i}} R_{i} \hat{\lambda}_{i}-A_{i} f_{i}=0 \rightarrow \\
\hat{\lambda}_{i}= & \left(Q_{y_{i}}+\left(A_{i}-\tilde{E}_{A_{i}}\right) \theta_{i}\left(A_{i}-\tilde{E}_{A_{i}}\right)^{T}+S_{i}^{T} Q_{\Phi_{i}} S_{i}\right. \\
& \left.+\left(A_{i}-\tilde{E}_{A_{i}}\right) \Sigma_{i-1}^{0}\left(\left(A_{i}-\tilde{E}_{A_{i}}\right)\left(\Phi_{i}-\tilde{E}_{\Phi_{i}}\right)\right)^{T}+\left(\left(\Phi_{i} \underline{x}_{i-1}+f_{i}\right)^{T} \otimes I_{m}\right) Q_{A_{i}} R_{i}\right)^{-1} \\
& \left(y_{i}-A_{i}\left(\Phi_{i} \underline{x}_{i-1}+f_{i}\right)\right)
\end{aligned}
$$

In the above equation, the inverse exists since the matrix $S_{i}$ is full column rank i.e. its quadratic form is invertible. After prediction of random observed variables $\tilde{e}_{i}, \tilde{e}_{A_{i}}, \tilde{e}_{\Phi_{i}}, \tilde{u}_{i}$ and $\tilde{e}_{i-1}^{0}$ iteratively using Eqs. (18)-(23), we must update the measured unknown parameters $\underline{x}_{i-1}$ and the corresponding dispersion matrix for the next epoch $i$. By applying variance propagation rules to Eq. (9), the updated dispersion matrix for the next epoch is given by 


$$
D\left(\underline{x}_{i}\right)=K_{i} \cdot \operatorname{blkdiag}\left(D\left(\underline{x}_{i-1}\right), D\left(e_{i-1}^{0}\right), D\left(u_{i}\right), D\left(E_{\Phi_{i}}\right)\right) \cdot K_{i}^{T}
$$

With

$$
K_{i}=\left[\begin{array}{llll}
\frac{\partial \underline{x}_{i}}{\partial \underline{x}_{i-1}} & \frac{\partial \underline{x}_{i}}{\partial e_{i-1}^{0}} & \frac{\partial \underline{x}_{i}}{\partial u_{i}} & \frac{\partial \underline{x}_{i}}{\partial E_{\Phi_{i}}}
\end{array}\right]=\left[\begin{array}{lll}
\left(\Phi_{i}-\tilde{E}_{\Phi_{i}}\right) & -\left(\Phi_{i}-\tilde{E}_{\Phi_{i}}\right) & I_{n}
\end{array}\right.
$$

$\left.-\left(\left(\underline{x}_{i-1}-\tilde{e}_{i-1}^{0}\right)^{T} \otimes I_{n}\right)\right]$

From Eq. (9) the update of the unknown parameters $\tilde{x}_{i}$ is obtained as follows:

$$
\tilde{x}_{i}=\left(\Phi_{i}-\tilde{E}_{\Phi_{i}}\right)\left(\underline{x}_{i-1}-\tilde{e}_{i-1}^{0}\right)+f_{i}+\tilde{u}_{i}
$$

Thus the update part for the next epoch is given by Eqs. (24) and (25). Summarizing, we propose the ITKF algorithm by the following flowchart:

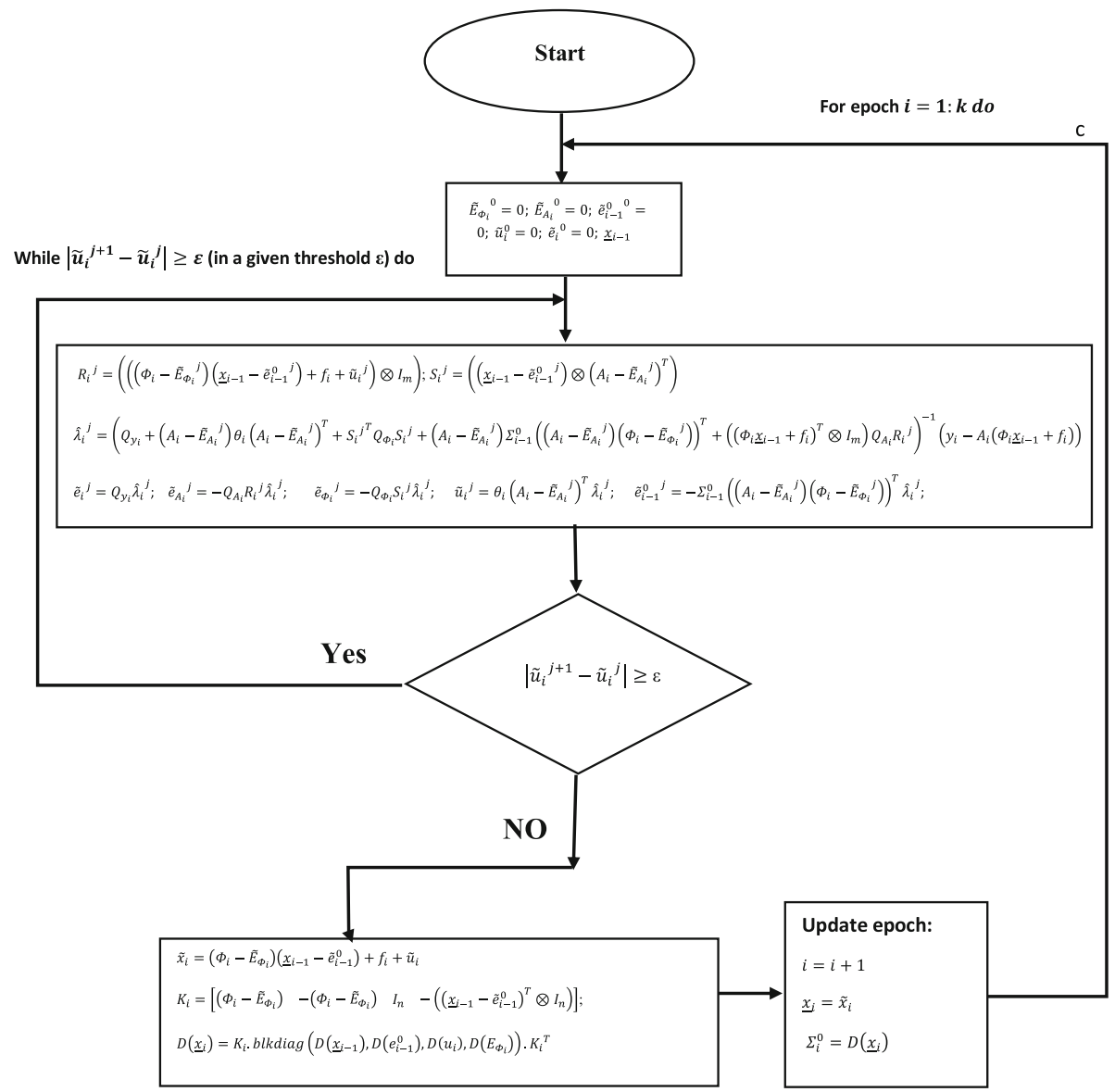




\section{ITKF algorithm for integrated direct geo-referencing}

If we want to produce the system equations by INS data for integrated direct geo-referencing, one has to consider Eq. (2) as the system equations where the coefficient matrix $\Phi_{i}$ is noisy i.e. $\underline{E}_{\Phi_{i}} \neq 0$. In order to sense this condition, we must examine the mathematical model of an INS system. It is obtained after solving navigation equations. For a background one may refer to Sheta (2012) or Jekeli (2001). Navigation equations are a set of differential equations which describe the input gyroscopes and accelerometers measurements input to the local frame mechanization and the output curvilinear coordinates, three velocity components, and three attitude components. Input gyroscopes are angular increments which are measured by IMU. Solving these vector differential equations, through integration, will result in a time variable state vector with kinematic sub-vectors for position, velocity, and attitude. The input to computation process are the angular increments measured by gyroscope and the velocity increments measured by accelerometer. The rotation matrix is updated by following Eq. (26). The Quaternion approach is used in the update because it deals with the singularity problems of the Euler angles at the 90 degrees angle. The quaternion is a 4 elements vector represented in space and contains the amplitude in one element and the direction is described using the three remaining elements. In general, the system equations can be described by the following equation

$$
\left[\begin{array}{c}
P_{i+1} \\
q_{i+1}
\end{array}\right]=\left[\begin{array}{cc}
I_{3} & 0 \\
0 & I_{4}+\underline{G}_{i}
\end{array}\right]\left[\begin{array}{c}
P_{i} \\
q_{i}
\end{array}\right]+\left[\begin{array}{c}
D_{i} \underline{V}_{i} \Delta t_{i} \\
0
\end{array}\right]
$$

where $\underline{G}_{i}=\frac{1}{2}\left[\begin{array}{cccc}\underline{c} & \underline{d} & -\underline{b} & \underline{a} \\ -\underline{d} & \underline{c} & \underline{a} & \underline{b} \\ \underline{b} & -\underline{a} & \underline{c} & \underline{d} \\ -\underline{a} & -\underline{b} & -\underline{d} & \underline{c}\end{array}\right], D_{i}$ is a deterministic matrix depends on radius of curvature, $\Delta t_{i}$ is time increments between two epochs and $P_{i}^{T}=\left[\begin{array}{lll}\varphi_{i} & \lambda_{i} & h_{i}\end{array}\right]$ is position and $q_{i}^{T}=\left[\begin{array}{llll}q_{1} & q_{2} & q_{3} & q_{4}\end{array}\right]_{i}$ denotes quaternion rotations. The noisy coefficients $\underline{a}, \underline{b}, \underline{c}$ and $\underline{d}$ are provided by the observed angular increments and the updated velocity $\underline{V}_{i}$ is produced by the observed velocity increments.

Consequently, the noisy coefficient matrix $\underline{\Phi}_{i}$, the unknown parameters $x_{i}$ and the vector $f_{i}$ introduced in Eq. (2) are as follows:

$$
\begin{gathered}
\underline{\Phi}_{i}=\left[\begin{array}{cc}
I_{3} & 0 \\
0 & I_{4}+\underline{G}_{i}
\end{array}\right] \\
f_{i}=\left[\begin{array}{c}
D_{i} \underline{V}_{i} \Delta t_{i} \\
0
\end{array}\right] \\
x_{i}=\left[\begin{array}{c}
P_{i} \\
q_{i}
\end{array}\right]
\end{gathered}
$$

Now suppose that for an integrated geo-referencing of a mobile sensor, we are going to determine the position and attitude of a mobile sensor at five epochs. Due to Eqs. (27)(29), the components of the DEIV model of the system equations at these epochs are as follows: 


$\Phi_{1}=\begin{array}{ccccccc}1 & 0 & 0 & 0 & 0 & 0 & 0 \\ 0 & 1 & 0 & 0 & 0 & 0 & 0 \\ 0 & 0 & 1 & 0 & 0 & 0 & 0 \\ 0 & 0 & 0 & 1.0413 & 0.3382 & -0.063321 & 0.10879 \\ 0 & 0 & 0 & -0.33916 & 1.0707 & 0.11561 & 0.061701 \\ 0 & 0 & 0 & 0.060071 & -0.085862 & 1.0615 & 0.33525 \\ 0 & 0 & 0 & 0.096412 & -0.060515 & -0.32074 & 1.0688\end{array}$

$\Phi_{2}=\begin{array}{ccccccc}1 & 0 & 0 & 0 & 0 & 0 & 0 \\ 0 & 1 & 0 & 0 & 0 & 0 & 0 \\ 0 & 0 & 1 & 0 & 0 & 0 & 0 \\ 0 & 0 & 0 & 1.0479 & 0.31649 & -0.037621 & 0.11486 \\ 0 & 0 & 0 & -0.3207 & 1.0434 & 0.1252 & 0.0826 \\ 0 & 0 & 0 & 0.062775 & -0.10455 & 1.0768 & 0.32767 \\ 0 & 0 & 0 & 0.094081 & -0.067946 & -0.30125 & 1.0621\end{array}$

$\Phi_{3}=\begin{array}{ccccccc}1 & 0 & 0 & 0 & 0 & 0 & 0 \\ 0 & 1 & 0 & 0 & 0 & 0 & 0 \\ 0 & 0 & 1 & 0 & 0 & 0 & 0 \\ 0 & 0 & 0 & 1.0654 & 0.3171 & -0.052309 & 0.093268 \\ 0 & 0 & 0 & -0.3152 & 1.0586 & 0.10666 & 0.071872 \\ 0 & 0 & 0 & 0.079853 & -0.10846 & 1.0636 & 0.3111 \\ 0 & 0 & 0 & 0.10865 & -0.07516 & -0.30661 & 1.047\end{array}$

$\Phi_{4}=\begin{array}{ccccccc}1 & 0 & 0 & 0 & 0 & 0 & 0 \\ 0 & 1 & 0 & 0 & 0 & 0 & 0 \\ 0 & 0 & 1 & 0 & 0 & 0 & 0 \\ 0 & 0 & 0 & 1.0565 & 0.32588 & -0.095492 & 0.10737 \\ 0 & 0 & 0 & -0.30382 & 1.0477 & 0.090393 & 0.064299 \\ 0 & 0 & 0 & 0.065948 & -0.096585 & 1.0389 & 0.32894 \\ 0 & 0 & 0 & 0.093003 & -0.060761 & -0.33936 & 1.0655\end{array}$

$\Phi_{5}=\begin{array}{ccccccc}1 & 0 & 0 & 0 & 0 & 0 & 0 \\ 0 & 1 & 0 & 0 & 0 & 0 & 0 \\ 0 & 0 & 1 & 0 & 0 & 0 & 0 \\ 0 & 0 & 0 & 1.0505 & 0.31213 & -0.09079 & 0.099056 \\ 0 & 0 & 0 & -0.31343 & 1.0605 & 0.090093 & 0.071849 \\ 0 & 0 & 0 & 0.061155 & -0.11065 & 1.0377 & 0.31949 \\ 0 & 0 & 0 & 0.091518 & -0.081141 & -0.33478 & 1.0527\end{array}$




$$
f_{1}=\left[\begin{array}{c}
1.9 \\
3.6 \\
2.2 \\
0 \\
0 \\
0 \\
0
\end{array}\right] ; f_{2}=\left[\begin{array}{c}
5.13 \\
8.9 \\
5.5 \\
0 \\
0 \\
0 \\
0
\end{array}\right] ; f_{3}=\left[\begin{array}{c}
2.79 \\
4.9 \\
3.12 \\
0 \\
0 \\
0 \\
0
\end{array}\right] ; f_{4}=\left[\begin{array}{c}
3.9 \\
7.1 \\
4.5 \\
0 \\
0 \\
0 \\
0
\end{array}\right] ; f_{5}=\left[\begin{array}{c}
3.3 \\
5.7 \\
3.4 \\
0 \\
0 \\
0 \\
0
\end{array}\right]
$$

For all of the DEIV models of these system equations, the stochastic model is given by

$$
\begin{gathered}
Q_{\Phi_{i}}=\left(I_{7} \otimes q\right)\left(I_{7} \otimes q\right)^{T} ; q=10^{-2}\left[\begin{array}{cccccccc}
0.6 & 0 & 0.4 & 0 & 0.1 & 0.2 & 0.1 \\
0 & 0.3 & 1 & 0.9 & 0.5 & 0.7 & 0 \\
0.6 & 0 & 1 & 1 & 0.2 & 1 & 0 \\
0.6 & 0.1 & 1 & 1 & 0.6 & 0.1 & 1 \\
0.2 & 0.3 & 0.4 & 1 & 0.6 & 0.1 & 0 \\
0.64 & 0.7 & 0.1 & 0.4 & 0.6 & 0.1 & 1 \\
0.1 & 0.3 & 0.5 & 0.4 & 0.3 & 0.02 & 1
\end{array}\right] ; \\
\theta_{i}=10^{-2}\left[\begin{array}{ccccccc}
2.96 & 3.4 & 1 & 0 & 0 & 0 & 0 \\
3.4 & 6 & 3.2 & 0 & 0 & 0 & 0 \\
1 & 3.2 & 2.44 & 0 & 0 & 0 & 0 \\
0 & 0 & 0 & 0 & 0 & 0 & 0 \\
0 & 0 & 0 & 0 & 0 & 0 & 0 \\
0 & 0 & 0 & 0 & 0 & 0 & 0 \\
0 & 0 & 0 & 0 & 0 & 0 & 0
\end{array}\right]
\end{gathered}
$$

Note that for $i=0,1,2, \ldots 6$ the $(7 i+1)$ to $(7 i+3)$ th. rows and columns of the matrix $\left(I_{7} \otimes q\right)$ must be replaced by zero.

The observation equations which can be produced by GPS and remote sensed data are given by 5 DEIV models at 5 epochs $i=1,2,3,4,5$ as

\begin{tabular}{ccccc}
\hline$y_{1}=$ & $y_{2}=$ & $y_{3}=$ & $y_{4}=$ & $y_{5}=$ \\
\hline 117.34 & 113.16 & 110.37 & 105.07 & 102.81 \\
158.14 & 151.1 & 145.48 & 136.77 & 132.9 \\
181.34 & 176.91 & 173.77 & 168.25 & 165.95 \\
604.6 & 462.26 & 332.86 & 206.12 & 93.749 \\
18.52 & 23.689 & 29.178 & 35.876 & 42.525 \\
-26.431 & -5.5668 & 18.249 & 40.574 & 68.688 \\
88.136 & 84.662 & 82.124 & 79.716 & 78.546 \\
681.14 & 520.54 & 373.63 & 229.62 & 101.29 \\
2466.6 & 1914 & 1409 & 911.46 & 470.96 \\
\hline
\end{tabular}




$\begin{array}{ccccccc}1 & 0 & 0 & 0 & 0 & 0 & 0 \\ 0 & 1 & 0 & 0 & 0 & 0 & 0 \\ 0 & 0 & 1 & 0 & 0 & 0 & 0 \\ 1.0094 & -0.08032 & -0.27102 & 1.3907 & 0.59041 & 0.16948 & 0.18762 \\ A_{1}=-1.1564 & -0.039561 & 0.9704 & 5.1452 & 2.3269 & 0.39127 & 0.0010988 \\ -2.334 & 1.4488 & 0.48338 & -2.6668 & 0.25197 & 0.075307 & 0.099471 \\ 1.4511 & -0.66802 & -0.011386 & -0.22686 & 2.2459 & 1.2784 & -0.2805 \\ 0.63161 & 0.11008 & -0.28957 & 2.4732 & 0.40092 & 0.18709 & -0.52125 \\ 3.7491 & 0.36467 & -0.23158 & -2.8492 & 2.2569 & 0.80019 & -0.19393\end{array}$

$\begin{array}{ccccccc}1 & 0 & 0 & 0 & 0 & 0 & 0 \\ 0 & 1 & 0 & 0 & 0 & 0 & 0 \\ 0 & 0 & 1 & 0 & 0 & 0 & 0 \\ 2.4707 & -0.099481 & -0.35429 & 1.5192 & 0.7432 & 0.11466 & 0.019044 \\ A_{2}=-1.0753 & -0.075316 & 0.99939 & 5.1984 & 4.5722 & 0.38824 & 0.032692 \\ -4.1786 & 2.7395 & 0.50222 & -4.6932 & -0.021168 & -0.02341 & -0.027835 \\ 1.636 & -0.73114 & 0.016827 & -0.0075877 & 2.0563 & 2.458 & -0.34538 \\ 2.6925 & 0.25707 & -0.044085 & 2.7252 & -0.005275 & 0.010675 & 0.02576 \\ 8.3343 & 0.53808 & 0.044516 & -2.284 & 1.7764 & 1.8865 & 0.12706\end{array}$

$\begin{array}{ccccccc}1 & 0 & 0 & 0 & 0 & 0 & 0 \\ 0 & 1 & 0 & 0 & 0 & 0 & 0 \\ 0 & 0 & 1 & 0 & 0 & 0 & 0 \\ 3.6732 & -0.10997 & -0.39623 & 1.615 & 0.9301 & 0.027076 & -0.025282 \\ A_{3}=-1.1203 & -0.17519 & 1.0344 & 5.2334 & 6.9007 & 0.39659 & 0.040885 \\ -6.2941 & 3.9875 & 0.72843 & -6.8312 & 0.022204 & -0.012321 & 0.27109 \\ 1.6144 & -0.81103 & 0.21158 & 0.038123 & 2.1286 & 3.8728 & -0.28363 \\ 3.2513 & -0.11541 & 0.098239 & 3.4351 & 0.083798 & -0.066311 & -0.045156 \\ 12.181 & 0.015706 & 0.37409 & -2.3089 & 2.1878 & 3.0923 & 0.33023\end{array}$

$\begin{array}{ccccccc}1 & 0 & 0 & 0 & 0 & 0 & 0 \\ 0 & 1 & 0 & 0 & 0 & 0 & 0 \\ 0 & 0 & 1 & 0 & 0 & 0 & 0 \\ 4.7735 & 0.061363 & -0.46255 & 1.5062 & 1.334 & -0.11011 & 0.34735 \\ A_{4}=-1.1271 & -0.19774 & 1.0138 & 5.1957 & 9.2138 & 0.36953 & 0.056945 \\ -8.5569 & 5.5994 & 0.64438 & -9.2217 & 0.2091 & 0.14771 & 0.35888 \\ 1.4718 & -0.67564 & 0.0097173 & -0.1208 & 2.1343 & 5.1686 & -0.19908 \\ 4.7702 & -0.0086341 & 0.097021 & 2.6353 & 0.35812 & -0.309 & 0.046051 \\ 16.055 & 0.28166 & 0.13936 & -2.2967 & 2.1511 & 3.7861 & 0.51141 \\ & & & & & & \\ 1 & 0 & 0 & 0 & 0 & 0 & 0 \\ 0 & 1 & 0 & 0 & 0 & 0 & 0 \\ 0 & 0 & 1 & 0 & 0 & 0 & 0 \\ 5.9639 & 0.03191 & -0.60776 & 1.4211 & 1.5153 & -0.04741 & 0.23179 \\ -1.1027 & -0.24537 & 0.97207 & 5.165 & 11.511 & 0.41599 & 0.043181 \\ -10.4 & 7.0747 & 0.61147 & -11.546 & 0.27357 & 0.15443 & 0.045528 \\ 1.6455 & -0.6405 & 0.033169 & -0.023809 & 2.2963 & 6.5331 & -0.31565 \\ 5.7747 & -0.059541 & -0.44762 & 2.462 & -0.14936 & 0.082947 & 0.14955 \\ 20.582 & 0.32071 & -0.12439 & -2.6433 & 2.4281 & 5.2551 & 0.43379\end{array}$

For all of the DEIV models of the observation equations, the stochastic model is given by 


$$
\begin{aligned}
Q_{x} & =\left(I_{7} \otimes q\right)\left(I_{7} \otimes q\right)^{T} ; q \\
& =10^{-1}\left[\begin{array}{ccccccccc}
1 & 0 & 0 & 1 & 0.1 & 0 & 0.4 & 0.6 & 0.2 \\
0.6 & 1 & 0 & 0 & 0.4 & 0 & 0.1 & 0.2 & 0.1 \\
1 & 0.3 & 1 & 0.9 & 0.5 & 0.7 & 0.1 & 0.2 & 0 \\
0.6 & 0 & 1 & 1 & 0.02 & 0.1 & 0 & 0.3 & 0 \\
0.6 & 0.01 & 0.1 & 0.02 & 0.1 & 0.1 & 0.06 & 0.1 & 0.1 \\
0.2 & 0.03 & 0.4 & 0.06 & 0.1 & 1 & 0.6 & 0.1 & 0 \\
0.4 & 0.07 & 0.1 & 0.04 & 0.2 & 0.4 & 0.6 & 0.1 & 0.1 \\
0.1 & 0.03 & 0.5 & 0.06 & 0.6 & 0.4 & 0.3 & 2 & 1 \\
0.6 & 0.01 & 1 & 0.06 & 1 & 1 & 0.6 & 0.1 & 1
\end{array}\right] ;
\end{aligned}
$$

For $i=0,1,2, \ldots 6$ the $(9 i+1)$ to $(9 i+3)$ th rows and columns of the matrix $\left(I_{6} \otimes q\right)$ must be replaced by zero.

$$
Q_{y}=10^{-4}\left[\begin{array}{ccccccccc}
69.06 & 9.78 & 33.69 & 16.73 & 9.15 & 5.58 & 29.6 & 5.53 & 9.11 \\
9.78 & 80.44 & -9.24 & 5.57 & 8.97 & 5.08 & 24.29 & 2.14 & 0.48 \\
33.69 & -9.24 & 57.78 & 2.28 & 23.12 & 5.43 & 33.16 & 18.54 & 12.08 \\
16.73 & 5.57 & 2.28 & 49.13 & 1.45 & 1.58 & 1.03 & 1.42 & 0.97 \\
9.15 & 8.97 & 23.12 & 1.45 & 37.34 & 1.172 & 2.07 & 4.68 & 5.32 \\
5.58 & 5.08 & 5.43 & 1.58 & 1.172 & 2.31 & 7.34 & 2.82 & 1.76 \\
29.6 & 24.29 & 33.162 & 1.03 & 2.07 & 7.34 & 117.3 & 43.52 & 6.88 \\
5.53 & 2.14 & 18.54 & 1.42 & 4.68 & 2.82 & 43.52 & 26.11 & 4.64 \\
9.11 & 0.48 & 12.08 & 0.97 & 5.32 & 1.76 & 6.88 & 4.64 & 3.91
\end{array}\right] \text {; }
$$

Also the observed state vector $x_{i}$ at an initial epoch with its corresponding dispersion matrix is given by:

$$
\sum_{0}^{0}=10^{-4}\left[\begin{array}{ccccccc}
4.01 & 0.4 & 0.1 & 0 & 0.0 & 0.0 & 0.0 \\
0.4 & 5 & 3 & 0 & 0.0 & 0.0 & 0.0 \\
0.1 & 3 & 2 & 0 & 0.0 & 0.0 & 0.0 \\
0.0 & 0.0 & 0.0 & 0.01 & 0.0 & 0.0 & 0.0 \\
0.0 & 0.0 & 0.0 & 0 & 0.01 & 0.0 & 0.0 \\
0.0 & 0.0 & 0.0 & 0 & 0.0 & 0.01 & 0.0 \\
0.0 & 0.0 & 0.0 & 0 & 0.0 & 0.0 & 0.01
\end{array}\right], x_{1}=\left[\begin{array}{c}
103.01 \\
132.9 \\
166 \\
-0.57 \\
-0.16 \\
-0 / 57 \\
0.56
\end{array}\right]
$$

In this problem both of the observation equations and system equations are in fact DEIV models. Three algorithms KF, TKF and ITKF are applied to this problem. We compare the result with true solution which are illustrated by Figs. 1 and 2 for 3-D position and attitude of the mobile sensor in a local frame respectively. The results demonstrated that the proposed ITKF approach can significantly improve the solution of the predicted position and attitude in contrast to other algorithms. Note that after computing the attitudes in quaternion representation, we converted them into three rotations about three axis in degrees. The improvement of the predicted position is more considerable than the predicted attitude. However, the TKF solution has larger difference with respect to true solution than the ITKF solution since it does not consider the random property of the random design matrix $\underline{\Phi}_{i}$. This situation gets worse for the KF solution in which not only we neglect the random property of the noisy design matrix $\underline{\Phi}_{i}$ but also the random design matrix $\underline{A}_{i}$ is considered deterministic i.e. with no noise. Moreover, the general treatment of 

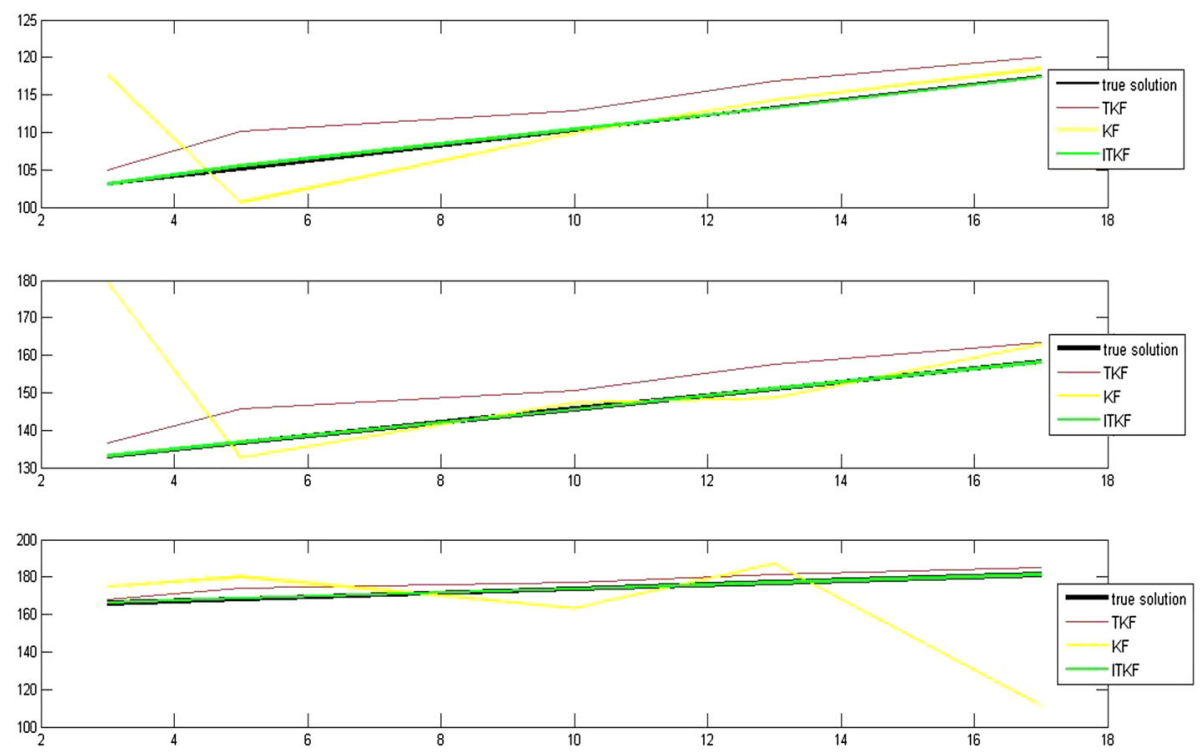

Fig. 1 solutions of different algorithms for 3-D position of the mobile sensor in a local frame
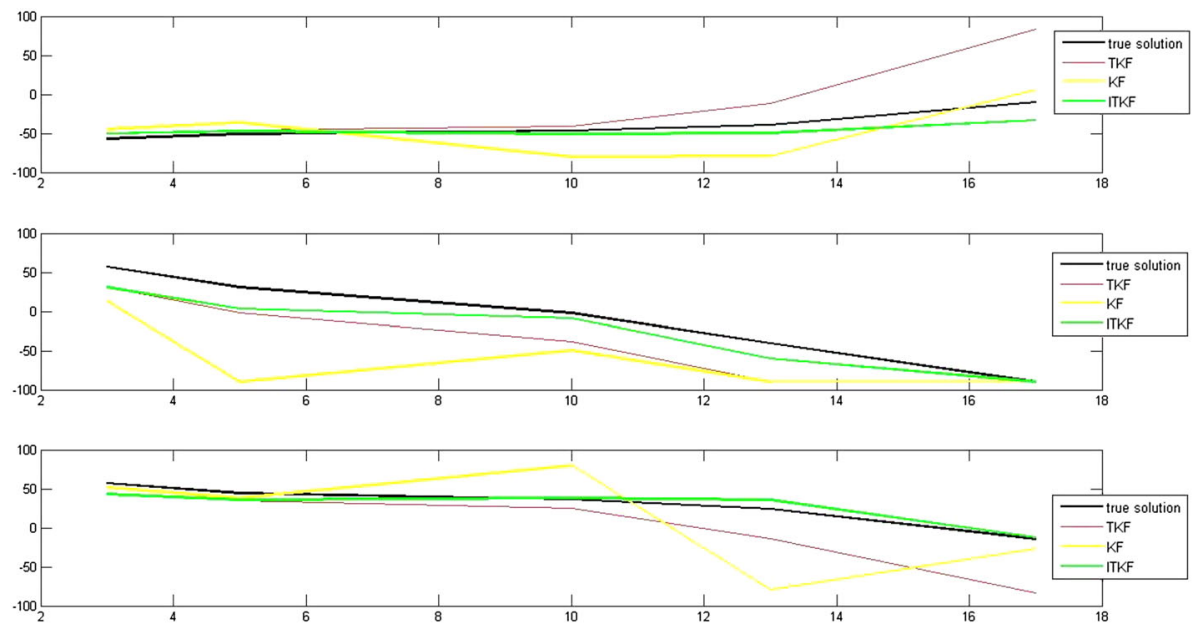

Fig. 2 solutions of different algorithms for 3-D attitude of the mobile sensor in a local frame

the TKF and ITKF approach are similar, however, we can see a significant bias in the TKF solution respect to the ITKF solution which is because of inappropriate modeling of the system equations made by the TKF approach, particularly when the magnitude of the weights of the elements in the random design matrixes $\underline{A}_{i}$ and $\underline{\Phi}_{i}$ cannot be neglected. 


\section{Conclusions and outlook}

In this paper, we developed a new Kalman filter algorithm. Its main assumption is that the system equations of a dynamic problem can itself be a DEIV model i.e. the design matrix $\underline{\Phi}_{i}$ of the system equations is also noisy. In practice one can see this situation when the system equations are provided by INS data. In such a case, the random noises are produced by observed angular increments and velocity increments. The predicted residuals for all variables besides the variance matrix of the unknown parameters were obtained by the proposed ITKF algorithm. In a numerical example, it was shown that the proposed ITKF approach can make the best improvement in solution in contrast to other algorithms, if both of the coefficient matrixes in the observation equations and the system equations are noisy. The prediction part is done by Eqs. (18)-(23) and the update part for the next epoch is given by Eqs. (24) and (25). In the forthcoming publication, we try to improve the prediction part due to several practical vulnerabilities of direct geo-referencing problem.

\section{References}

Amiri-Simkooei A, Jazaeri S (2012) Weighted total least squares formulated by standard least squares theory. J Geod Sci 2(2):113-124

Evensen G (1994) Sequential data assimilation with a nonlinear quasigeostrophic model using Monte Carlo methods to forecast error statistics. J Geophys Res 99(C5):10143-10162

Fang X (2011) Weighted total least squares solutions for applications in geodesy. Ph.D. thesis.Leibniz University, Hannover

Fang X (2013) Weighted Total Least Squares: necessary and sufficient conditions, fixed and random parameters. J Geod 87(8):733-749

Fang X (2014a) On non-combinatorial weighted total least squares with inequality constraints. J Geod 88(8):805-816

Fang X (2014b) A structured and constrained total least-squares solution with cross-covariances. Stud Geophys Geod 58(1):1-16

Fang X (2014c) A total least squares solution for geodetic datum transformations. Acta Geod Geophys 49(2):189-207

Fang X (2015) Weighted total least-squares with constraints: a universal formula for geodetic symmetrical transformations. J Geod 89(5):459-469

Fang X, Wu Y (2015) On the errors-in-variables model with equality and inequality constraints for selected numerical examples. Acta Geod Geophys. doi:10.1007/s40328-015-0141-5

Fang X, Wang J, Li B, Zeng W, Yao Y (2015) On total least squares for quadratic form estimation. Stud Geophys Geod 59(3):366-379. doi:10.1007/s11200-014-0267-x

Fang X, Li B, Alkhatib H, Zeng WX, Yao YB (2016) Bayesian inference for the Errors-In-Variables model. Stud Geophys Geod. doi:10.1007/s11200-015-6107-9

Gelb A (1974) Applied optimal estimation. MIT Press, Massachusetts

Jazwinski AH (1970) Stochastic process and filtering theory. Academic Press, New York

Jekeli C (2001) Inertial navigation systems with geodetic applications. deGruyter, Berlin/New York

Julier SJ, Uhlmann JK (1997) A new extension of the Kalman filter to nonlinear systems. In: Proceedings of the SPIE aerosense international symposium on aerospace/defense sensing, simulation and controls, Orlando, Florida

Kalman RE (1960) A new approach to linear filtering and prediction problems. Trans ASME Ser D J Basic Eng 82:34-45

Lefebvre T, Bruyninckx H, De Schutter J (2002) Comment on "a new method for the nonlinear transformation of means and covariances in filters and estimators". IEEE Trans Autom Control 47(8):477-482

Liu J, Chen R (1998) Sequential Monte Carlo methods for dynamic systems. J Am Stat Assoc 93:1032-1044

Lu J, Chen Y, Li BF, Fang X (2014) Robust total least squares with reweighting iteration for threedimensional similarity transformation. Surv Rev 46(334):28-36

Mahboub V (2012) On weighted total least-squares for geodetic transformations. J Geod 86:359-367. doi:10.1007/s00190-011-0524-5 
Mahboub V (2014) Variance component estimation in errors-in-variables models and a rigorous total least squares approach. Stud Geophys Geod 58:17-40. doi:10.1007/s11200-013-321150-x

Mahboub V (2016) A weighted least-squares solution to a 3-D symmetrical similarity transformation without linearizationStud. Geophys Geod 60:1-30. doi:10.1007/s11200-014-1041-9

Mahboub V, Sharifi MA (2013a) On weighted total least-squares with linear and quadratic constraints. J Geod 87:279-286. doi:10.1007/s00190-012-0598-8

Mahboub V, Sharifi MA (2013b) Erratum to: on weighted total least squares with linear and quadratic constraints. J Geod 87:607-608

Mahboub V, Amiri-Simkooei A, Sharifi MA (2012) Iteratively reweighted total least-squares: a robust estimation in errors-in-variables models. Surv Rev 45:92-99. doi:10.1179/1752270612Y.0000000017

Mahboub V, Ardalan AA, Ebrahimzadeh S (2015) Adjustment of non-typical errors-in37 variables models. Acta Geod Geophys 50:207-218. doi:10.1007/s40328-015-0109-5

Mahboub V, Saadatseresht M, Ardalan A (2016) A general weighted total Kalman filter algorithm with numerical evaluationStud. Geophys Geod, 61 (2017), doi: 10.1007/s11200-016-0815-7 (in print) ic 2016 Inst. Geophys CAS, Prague

Maybeck P (1979) Stochastic models, estimation, and control, vol 1. Academic Press, Waltham, republished 1994

Neitzel F (2010) Generalization of total least-squares 1 on example of unweighted and weighted similarity transformation. J Geod 84:751-762

Neitzel F, Schaffrin B (2016) On the Gauss-Helmert model with a singular dispersion matrixwhere BQ is of smaller rank than B. J Comput Appl Math 291(2016):458-467. doi:10.1016/j.cam.2015.03.006

Paláncz B, Awange L (2012) Application of Pareto optimality to linear models with errors-in-all-variables. J Geod 86(7):531-545

Sanso F (1986) Statistical methods in physical geodesy. In: Sunkel H (ed) Mathematical and numerical techniques in physical geodesy. Lecture notes in earth sciences, vol 7. Springer, Berlin, pp 49-155

Schaffrin B, Felus Y (2008) On the multivariate total least-squares approach to empirical coordinate transformations. Three algorithms. J Geod 82:353-383

Schaffrin B, Iz HB (2008) Towards total Kalman filtering for mobile mapping. Int Arch Photogramm Remote Sens Spat Inf Sci 36, Part 5/C55:270-275

Schaffrin B, Uzun S (2011) Errors-In-Variables for mobile mapping algorithms. In: The presence of OutIIERS, archives of photogrammetry, cartography and remote sensing, Vol. 22, pp 377-387, ISSN 2083-2214

Schaffrin B, Snow K, Neitzel F (2014) On the Errors-In-Variables model with singular dispersion matrices. J Geod Sci 4:28-36. doi:10.2478/jogs-2014-0004

Shen YZ, Li BF, Chen Y (2011) An iterative solution of weighted total least squares adjustment. J Geod $85: 229-238$

Sheta B (2012) Vision based navigation (VBN) of unmanned aerial vehicles (UAV), Ph.D. thesis, Calgary, Alberta

Skaloud J (1999) Problems in direct-georeferencing by INS/DGPS in the airborne environment. In: Invited paper, ISPRS workshop on 'direct versus indirect methods of orientation' WG III/1, Barcelona, 25-26 November 1999

Snow K, Schaffrin B (2012) Weighted Total Least-Squares collocation with geodetic applications. In: SIAM conference in applied linear algebra, Valencia, Spain

Sorenson HW (1966) Kalman filtering techniques. In: Leondes CT (ed) Advances in control systems: theory and applications, vol 3. Academic press, New york, pp 219-292

van der Merwe R, Wan EA (2003) Sigma-point Kalman filters for probabilistic inference in dynamic statespace models. In: Workshop on advances in machine learning, Montreal

Welch G, Bishop G (2001) An introduction to the Kalman filter, SIGGRAPH 2001, Los Angeles, CA, August 12-17, 2001

Yi Y (2007) On improving the accuracy and reliability of Gps/INS-based direct sensor georeferencing. PhD thesis, The Ohio State University

Zeng W, Liu J, Yao Y (2015) On partial errors-in-variables models with inequality constraints of parameters and variables. J Geod 89(2):111-119

Zhang S, Tong X, Zhang K (2013) A solution to EIV model with inequality constraints and its geodetic applications. J Geod 87(1):23-28. First online: 13 June 2012

Zhou Y, Fang X (2015) A mixed weighted least squares and weighted total least squares adjustment method and its geodetic applications. Surv Rev. doi:10.1179/1752270615Y.0000000040 